\title{
Sachregister zu Band 65
}

Die fett gedruckten Zahlen bezeichnen Eigenarbeiten. Bb. = Buchbesprechung.

A Ablatio retinae 363 Absaugevorrichtung zur Trok-

kenhaltung des Operationsfeldes

358 Adaptation bei Glaukom 108 Adenotomie bei Augenaffektionen

293 Aderhaut s. Chorioidea Agraf 288 Alkoholmißbrauch, Punktion der

vorderen Rammer bei Amblyopie

infolge von - 294 Amblyopia ex anopsia 261 Amblyopie, Punktion der vorderen

Kammer bei - infolge Alkohol-

und Nikotinmif3brauchs 294 Ammoniakverätzung mit gün-

stigem Ausgang 123 Angiofibrom der Orbita 89 Angiopathien der Netzhaut 281

- $\quad$ traumatische 375Anisokorie, temporäre - bei einer

Leprakranken 122 Aphakie, Binokularsehen bei ein-

seitiger - 199 Arachnoiditis, Symptome ver-mehrten Hirndruckes infolge uni-

schriebener - 121 Arteria centralis retinae, Pseu-

doembolie der - 106 Asthenopie, ein seltener Fall von

- $\quad 368$

Atophanyl, Heilung einer Ophthalmia sympathica durch - 202 Auge, Entwicklung des menschlichen - 319 (Bb.) Augenarzt als Kriminalist 198 Augenbewegungen, einseitige

willkürliche - 274 Augenheilkunde 125 (Bb.) Augenhintergrund, Befunde am

- $\quad$ bei leptomeningealen Spontan-blutungen 363

- $\quad$ strangartige Bildung am - 356Augenlider s. LiderAugenmuskeln, angeborene Be-

weglichkeitsstörungen der -1

Erkrankungen der - 258ff.

Phlegmone der äußeren - 200 Augenschutzgläser, die physiolo-

gischen Grundlagen und die phy-sikalischen Eigenschaften der wichtigsten - 371

Azetylenbetrieb, Ätzungen im -

124

B Bakterienfiltrate, die keimíreien-

nach Besredka und ihre thera-

peutische Anwendung 135 Basalzellenepitheliom der Lider

90 Belichtung und Verdunklung,

Einfluß der - auf den Augen-

druck 107 Benzolfabrik, eigenartiger Unfall

in einer - 383 Besredkasche Bakterienfiltrate 135 Bindehaut s. Conjunctiva Binokularsehen bei einseitiger

Aphakie 199 Blepharochalasis 90 Blick, der gesenkte - no, 244 Blickfeld, das praktische - Einäugiger 276Blicklähmungen 363Blinde, Topographie der im

Kiewer Bezirk 118 Blindenstatistik in Köln 200 Blindenwesen 384 Blitzkatarakt 104

Brillengläser, Wege zur Bestim- 
mung der Scheitelrefraktion von

- $\quad$ 358Brillenlehre 208 (Bb.)

Bulb us, Stellung des - bei ge-

schlossenem Auge no, 251 Bulbuskompression, Folgen der

- $\quad$ bei Raumbeengung in derOrbita 108

C Candiolin Bayer 290 Caruncula lacrymalis, Polypen

der - 93 Chiasma, Schußverletzung des -

314

Chiffres de $\Gamma$ lris 344 Chlorakne 316 Cholesterinkristalle in der Linse

118 Choleval 292 Chorioidea, Tumoren der - 99,

100

Verknöcherung der - 99

zirkumpapilläre Atrophie der -bei My open 99

$390 \quad$ Sachregister zu Band 65.

Conjunctiva, Pathologie der 90if.

primäre Tuberkulose der -117

Sensibilität der - 203 Conjunctivitis, Diphtherieserum

bei pseudomembranöser, diphthe-risc£ter und ekzematöser - 291

ble nn orrhoica, Gonokokken-vakzine bei - 293

gonorrhoica, Kokkenbefundbei metastatischer - 299

neonatorum, Prophylaxe der

- 290

purulenta, Vakzinebehandlung der -292

vernalis, Natriumthiosulfatge-gen -375

Cornea, Pathologie der - 94if. - - -empfindlichkeit gegen Druck-ausübung am Lide, Prüfung der

- $\quad$ als diagnostisches Hilfsmittel188

geheiltes Karzinom der - 375

knötchenförmigeVeränderung der Rückfläche der - bei Groenouw-scher familiärer

Hornhautdystro-phie 240

Kristalle in der -357

-naht, prophylaktische - bei Staroperationen 361

Sensibilität der -203

- experimentelle Untersuchun-gen über die - mittels der Re-aktionszeitmethode 63

- $\quad$ Xanthomatose der - 366Corpus ciliare, Modifikation der

Struktur des - infolge von Glaukomoperationen 100

- $\quad$ Sarkom des -310

Corpus vitreum, Entfernung ma-gnetischer Fremkörper aus dem

- $\quad$ 288Cyklodialyse 372Cysticercus subretinalis 309Cysticercusoperation 310

T)

Dakryocystitis, chirurgische Be-handlung der - 291, 292

Diphtherieserum bei pseudomembranöser, diphtherischer und ekzematöser Conjunctivitis 291

Druck, intraökulärer, Einfluß der Verdunklung und Belichtung auf den - 107

Druckspülung, eineinfacher Hand-griff zur Ausführung der - bei Verengerung der Tränenkanäle 83

E Eigenblutserum, Behandlung des Trachoms mit - 93, 293

Einäugige, das praktische Blick-feld der - 276 
- $\quad$ Kraftfahrer 123Elliotsche Trepanation, doppel-

seitige Infektion nach - mit Heilung 369 Entropium mit Trichiasis, Operation bei - 294

- Operationsmethoden gegen Nar-ben - 376

Ephetonin 306

Epipapilläre Bildungen und Mem-

branen 382 Exophthalmus, intermittierender

- $\quad$ infolge von Varizen der Orbita290

Epiphora, atonische - 376 Exophthalmus als Folge einer Zahnextraktion 309

$\Gamma$ Feuerarbeiter, professionelle Lin-

sentrübungen bei - in Fiebertherapie bei Keratitis pa-

renchymatosa 311

- $\quad$ verschiedener Augenleiden mitPyrifer 10

Fleischerscher Ring bei Wilson-

scher Krankheit 114 Fremdkörper, durch das rechte

Auge in die Schädelkapsel ein-

gedrungener Mantelsplitter eines

Artilleriezünders 308

- $\quad$ Entfernung magnetischer - ausdem Glaskörper 288

- $\quad$ Holzsplitter im Auge 353Furunkel, Orbitalphlegmone als

Folge eines - der Wange in

a

Gehirndruck, Symptome von ver-mehrtem - infolge umschriebe-ner Arachnoiditis 121

Gehirnkrankheiten, reflektori-sche Pupillenstarre und Ptosis als Oberflächensymptome bei

basalen, vorwiegend luetischen

- 203

Gehirnstein in der suprasellaren

Gegend als Ursache einer Seh-

nervenatrophie 192 Gehirntumoren und Stauungs-

papille 121 Gesenkter Blick no, 244 Gesichtsfeldprüfungen während

der letzten Wochen der Schwan-

gerschaft 120 Glasbläserstar 205 Glaskörper s. Corpus vitreum Glaukom, Adaptation bei - 108

Sachregister zu Band $65 . \quad 391$

Glaukom, Einfluß der Verdunk-lung und Belichtung auf den Druck bei - 107

-behandlung 373

-operationen 288

Modifikation der Struktur des Corpus ciliare infolge von - 100

Gleichgewicht und Gleichge-wichtsstörungen 271

Glioma retinae, Netzhauttuber-kulose unter dem Bilde eines - 198

Goldbehandlung bei tuberkulösen Augenkrankheiten 312

Gonokokkenvakzine bei Conjunctivitis blennorrhoica 293

Groenouwsche familiäre Horn-hautdystrophie, knötchenför-mige Veränderung der Hornhaut-

rückfläche bei -240

Gynergen bei intraokularer Druck-steigerung 108

$\mathrm{H}$

Hämangioendotheliom desOber-lides und der Orbita im Säug-lingsalter 150

Heilpädagogik des Auges 386 (Bb.) 
Herpes und Auge 121

- $\quad$ corneae febrilis in der Unfall-ophthalmologie 317

Hertwig-Magendiesches Phä-

nomen 262 Heterochromie mit einseitiger Ka-

tarakt 117 v. Hippelsche Krankheit 191 Höllensteinlösung, Püretten für

die - 122 Holzsplitter im Auge 3õ3 Hornhaut s. Cornea Hypophysis, Augenerscheinungen

bei Erkrankungen der - 289

I.

Immunität, lokale erworbene - der orbitalen Gewebe 89

Immuntherapie der Augenkrankheiten 294

Iontophorese 293

- $\quad$ in der Behandlung der Katarakt104

Iridektomie, Blutung in die Linse nach - 295

- $\quad$ periphere - bei der Staroperation291

iridocyclitis tuberculosa, thera-peutische Wirkung des Serums Trachomkranker bei - 292

- $\quad$ Verlötungsvorgang am Kammer-winkel bei - 303

Iris, Chiffres de $\Gamma$ lris 344

Iriskolobom, beiderseitiges - bei eineiigen Zwillingen 98

Sarkom der -310

traumatische Zysten der - 97

-Tuberkulos $\cdot e$, Behandlung der experimentellen - mit ultra-violetten Strahlen Ill

$\mathrm{J}$

Jahrbuch der Ophthalmologie, amerikanisches $126(\mathrm{Bb}$.

$\mathrm{K}$

Kammer, vordere s. Vorder-kammer

Kammerwasser, Veränderungen der Linsenfasern infolge wieder-holter Entleerungen des - 103

Kammerwinkel, Verlötungsvorgang des - bei chronischer Iridocyclitis 303

Karzinom, geheiltes - der Hornhaut 375

Katarakt 102, 104

familiäre $\cdot$ Rettungsgürtel -198

Glasbläser - 205

juvenile mit großen Kernen bei zwei geheilten Tuberkulösen 207

Strahlen - 303 Kataraktoperation 292, 293

Kollaps der Sklera bei der - 289

Nähte bei der -310

periphere Iridektomie bei der -291

prophylaktische Hornhautnaht bei - 361

Keratitis, zentrale sklerosierende

- 372 - parenchymatosa, Fiebertherapie bei - 311

- Wismutbehandlung der -312 Keratoconj unctivitis ekzema-

tosa, Pathogenese der - 92 Keratokonus, chirurgische Behandlung des - 293 Keratoplastik,

Technik der par-

tiellen durchgreifenden - 147 Knochenbildung in der Aderhaut 
99 Konvergenzkrampf 274 Körperbau und Refraktion 378 Kraftfahrer, einäugige 123

Kristallbildungen in der vorderen

Augenkammer und Linse 219

- $\quad$ in der Hornhaut 357Krönleinsche Operation bei Seh-

nervengeschwulst 119 Kupfer zu parenteralen Injektionen bei Trachom 291

L

Labyrinth und Schielen 14

392 Sachregisier

zu Band 65.

Lepra, temporäre Anisokorie bei -

122 Leptomeningeale Spontanblu-

tungen, Augenhintergrundsbe-

funde bei - 363 Lichttherapie des Auges 391 (Bb.),

349 Li der, Erkrankungen der - 90

Lidwinkelplastik 382

Naevus des -112

Plastik am unteren - nach Bü-dinger 310

Stellung des - bei geschlossenem Auge no, $2 \delta 1$

- $\quad \backslash$ terruköse Tuberkulose der - 208Linse, Blutung in die - nach Glau-

komiridektomie 295

Cholestearinkristalle in der -118

Kristallbildungen in der -219

Pathologie der - loiff.

reklinierte - 304 Linsenatmung 41 Linsenkapsel, Gefäße auf der vor-

deren - - in der Pupille ohne

merkliche Schwartenbildung 129 Linsenluxation 102, 103 -- geheilter Fall einer subkonjunk-

tivalen - 375 Linsentrübungen, professionelle

- bei Feuerarbeitern in Lues, Form der Pupille bei - 97

- $\quad$ Soziologie der - 200Lymphadenose 305

M Maculaaussparung 112 Maculadegeneration, hereditäre

no - senile 279 Megalocornea 356 Membranapupillarispersistens

corneae adhaerens, eine Wim-

per in der vorderen Kammer vor-

täuschend 346 Meningitis luetica, plötzlicher

Tod und Unfall 124 Metastasenbildungen 304 Methylalkoholvergiftung mit

konsekutiver Blindheit und Exi-

tus letalis 237 Migraine ophtalmique asso-

ciée 304 Mikroskopie des lebenden Auges

an der Gullstrandschen Spalt-

lampe 385 (Bb.) Mißbildung, angeborene - der

Augen 118 Musculusobliquus inferior dexter, Torticollis infolge angebo-

rener Überfunktion des - 106

Myektomie des Obliquus inferior

260 Myopie, zirkumpapilläre Chorioi-

dealatrophie bei - 99 
N Naevus des Augenlids 112

- melanotischer- der Conjunctiva

9i, 93

Narbenentropium, Operations-methoden gegen - 376

Natriumthiosulfat gegen Conjunctivitis vernalis 375

Nervus opticus, Erkrankungen des - $278 \mathrm{ff}$.

Krönleinsche Operation bei Ge-schwulst des - 119

-atrophie infolge Hirnsteins in der suprasellaren Gegend 192

- rhinologische operative Be-handlung der - 194, 33(5

Netzhaut s. Retina Neuritis als Folge einer Zahn-extraktion 309

- $\quad$ retrobulbaris 296Neurologie des Auges $385(\mathrm{Bb}$.)Neuroma plexiforme palpebrae

90 Nik·otinmißbrauch, Punktion der

vorderen Kammer bei Amblyopie

infolge von - 294 Nystagmus 262ff.

- monocularis 162

- $\quad$ Nachweis von Sehstörungen mit-tels des optischen Dreh - 197

0

Ophthalmia sympathica, durch

Atophanyl geheilt 202 Ophthalmologische Einrich-

tung, tragbare -375 Ophthalmoplegia internuclea-

ris 274 Optik 282

Optikus s. Nervus opticus Optisch-motorisches Feld und

optisch-motorische Bahn 267 Orbita, Erkrankungen der - 88, 89

- $\quad$-plastik 375Orbitalphlegmone als Folge eines

Wangenfurunkels in

$P$

Paraffinom am Oberlid nach kos-

metischer Paraffininjektion in der

Wangengegend 298 Parazentese der Vorderkammer

291 Papillome der Conjunctiva 93 Pemphigusconj unctivae, Histo-

genese des - 91 Pernakr ankheit 316

Sachregister zu Band 65.

393

Pilzkonkremente, Affektion der

Tränenwege durch - 79 Phlegmon e der äußeren Augen-

muskulatur 200 Phlyktaene, diagnostische Bedeu-

tung der - 367 Plastik am Unterlide nach Bü-

dinger 310

Lidwinkel - 382

Orbita -- 375

Poly pen der Caruncula lacrymalis

und der Conjunctiva 93 Prisoptometer 309 Prothesen, Herstellung provisori-

scher - 256 Ptosis als Oberflächensymptom bei

basalen, vorwiegend luetischen

Gehirnkrankheiten 203 Pupille, Form der - bei Syphilis 
springende 115

Pupil lenbewegung, Mechanismus

der - 98 Pupillenenge, traumatische maxi-

male 329 Pupillenphänomene, eigenartige - bei angeborener Oculomoto-

riuslähmung 273

- $\quad$ zur Kasuistik wechselnder - 82Pupillenstarre, reflektorische

als Oberflächensymptome bei basalen, vorwiegend luetischen Gehirnkrankheiten 203

Pupillenstörungen und Lebens-dauer 166

Püretten für die Höllensteinlösung 122

Pyrifer, Fieberbehandlung ver-schiedener Augenleiden mit - 10

$\mathrm{R}$

Radiotherapie der Sarkome der Nebenhöhlen und der Orbita 287

- Internationale - $386(\mathrm{Bb}$.)Raumbeengung in der Orbita,

Folgen der Bulbuskompression

bei - 108 Reaktionszeitmethode, experi-

mentelle Untersuchungen über

die Hornhautsensibilität mittels

der - 63 Refraktion und Körperbau 378 Refraktionslehre 208 (Bb.) Retina, Angiopathia r.

traumatica

375

-blutungen, massige -- 381

Erkrankungen der - 279ft.

scheibenförmige Entartung der Mitte der - 193, 299, 379

-tuberkulose unter dem Bilde eines Glioma retinae 198

Retinitis pigmentosa, ein neuer

Weg zur Behandlung der - 189 Retinitis punctata albescens

279 Rettungsgürtelkatarakt. fami-

liäre - 198 Röntgenbehandlung der Augen-

krankheiten 306, 317 (Bb.)

- $\quad$ bei den Affektionen der tiefenMembranen des Bulbus 293

Röntgenstrahlen, Retinitis pigmentosa durch - 280

- Wirkung der - auf die Linsen-fasern 104

Rotgrünblindheit, Erblichkeits-verhältnisse bei den verschiedenen Arten der - 119

S Salvarsan, Technik bei Injektion

von - 381 Sarkoid Boeck und Auge 71 Sarkom der Iris und des Corpus

ciliare 310 - der Orbita, operiert nach Cirin-

cione 360

- $\quad$ Radiotherapie der - der Nebenhöhlen und der Orbita 287

Säugetier- und Menschenauge

no Säure-Alkohol-Wirkung bei den

Tintenstiftverletzungen des Auges

366 Scheitelbrechwertmessung in

der Praxis 201 Schielen 258ft.

- $\quad$ und Labyrinth 14

zentrales Skotom bei - in Schulen für Sehschwache 305 
Leitsätze betreffend die - 195 Schwangerschaft, Gesichtsfeld-

prüfungen während der letzten Wochen der -- 120 Schweißer, Augenschädigungen der

- 313 Sehschwachenschule 305

- $\quad$ Leitsätze betreffend die - 195Sehstörungen, Nachweis von -

mittels des optischen Dreh-

nystagmus 197 Sensibilität von Cornea und Conjunctiva 203 Siderosis 199 Ski era, Kollaps der bei der Ka-

taraktoperation 289 Skotom, zentrales - bei Schielen-

den in Spaltlampe, Mikroskopie des le-

benden Auges an der Gullstrand-

schen - 385 (Bb.) Sporillose der Conjunctiva 92

394 Sachregister zu Band 65.

Springende Pupillen 115 Stauungspapille und Hirntumo-

Ten 121 Sterilisation, trockene - der In-

strumente 358 Strabismus s. Schielen Strahlenkatarakt 303 Strahlungen, Grenzintensitäten iür

die Erträglichkeit der - 371 Streptothrix, Affektion der Trä-

nenwege durch - 79 Sympathische Entzündung und

sympathische Reizung 24 Synchisis scintillans camerae

anterioris 85

$\mathrm{T}$

Tätowierung, chemische - (mit

Silber) 309 Tebeprotin bei Augentuberkulose

312 Teratome, zystische - der Orbita

88 Tintenstiftverletzungendes Au-

ges, Säure-Alkohol-Wirkung bei

den - 366 Tonometer, Kontrolle des Schiötz

- $\quad 372$

Totische Operation 119 Torticollis infolge angeborener Überfunktion des Obliquus inferior dexter 106 Trachom 94, 294

Behandlung des - 92, 93

Eigenblutbehandlung bei -293

Kupfer zu parenteralen Injek-tionen bei - 291

neuere Anschauungen über das

- $209 \quad 1$

- $\quad$ therapeutische Wirkung des Serums kranker bei tuberkulöser

Iridocyclitis 292

Verhalten der Tränenwege beim

$-\quad 282$

Tränenkanäle, eineinfacher Hand-griff zur Ausführung der Druck-spülung bei Verengerung der 83

Affektion der - durch Püz-konkremente 79

gonorrhoische Erkrankung der - 301

Verhalten der - beim Trachom 282

Tuberkulose der Cornea 95

Goldbehandlung und Tebeprotin bei Augen- - 312

juvenile Katarakt mit großen Kernen bei zwei geheilten Tuber-kulösen 207 
primäre - der Lidbindehaut 117

und Trachom 94

verruköse - der Lider 208

U Ultraviolette Strahlen, Behandlung der experimentellen Iris-tuberkulose mit - in

- $\quad$ Behandlung von Augenkrank-heiten mit - 232, 310

Unfallkunde 123, 124, 3i2Íf., 383 Uvea, Erkrankungen der - 98 ff.

$\mathrm{V}$

Vakzinebehandlung der Conjunctivitis purulenta neonatorum 292, 293

- $\quad$ bei Erkrankungen der Tränenwege und des Bulbus 293

Varizender Orbita als Ursache eines

intermittierenden Exophthalmus

290 Verdunklung und Belichtung,

Einfluß der - auf den Augen-

druck 107 Versicherungskunde 123, 124,

3121I, 383 Vierhügeltumor 113 Vorderkammer, freies bewegliches

Bläschen in der $\mathbf{-}-96$

Kristallbildungen in der -219

Parazentese der - 291

postoperative Epithelauskleidung der - 198

Punktion der - bei Amblyopie infolge Alkohol- und Nikotin-mißbrauchs 294

Synchisis scintillans der -85

Technik der Eröffnung der - bei aufgehobener Kammer 105

W

Wilsonsche Krankheit, Flei-scherscher Ring bei - 114

Wismutbehandlung der Augen-syphilis 286, 292

- $\quad$ der Keratitis parenchymatosa 312

$\mathrm{X}$

Xanthomatose der Hornhaut 366

Z

Zahnextraktion, Neuritis und Exophthalmus als Folge einer - 309

Zyste, seröse - der Orbita, von ver-sprengter Nasenschleimhaut aus-gehend 321

-. - $\quad$ traumatische - der Iris 97Zwillinge

ophthalmologische Un-

tersuchungen bei ein- und zwei-eiigen -202 